\title{
Percutaneous Drainage versus Emergency Cholecystectomy for the Treatment of Acute Cholecystitis in Critically Ill Patients: Does it Matter?
}

\author{
E. Melloul $\cdot$ A. Denys $\cdot$ N. Demartines $\cdot$ \\ J.-M. Calmes • M. Schäfer
}

Published online: 12 February 2011

(C) Société Internationale de Chirurgie 2011

\begin{abstract}
Background The aim if this study was to compare percutaneous drainage (PD) of the gallbladder to emergency cholecystectomy (EC) in a well-defined patient group with sepsis related to acute calculous/acalculous cholecystitis (ACC/AAC).

Methods Between 2001 and 2007, all consecutive patients of our ICU treated by either PD or EC were retrospectively analyzed. Cases were collected from a prospective database. Percutaneous drainage was performed by a transhepatic route and EC by open or laparoscopic approach. Patients' general condition and organ dysfunction were assessed by two validated scoring systems (SAPS II and SOFA, respectively). Morbidity, mortality, and longterm outcome were systematically reviewed and analyzed in both groups.

Results Forty-two patients [median age $=65.5$ years (range $=32-94)]$ were included; $45 \%$ underwent EC (ten laparoscopic, nine open) and 55\% PD $(n=23)$. Both patient groups had similar preoperative characteristics. Percutaneous drainage and EC were successful in 91 and $100 \%$ of patients, respectively. Organ dysfunctions were similarly improved by the third postoperative/postdrainage days. Despite undergoing PD, two patients required EC due to gangrenous cholecystitis. The conversion rate after laparoscopy was $20 \%$. Overall morbidity was $8.7 \%$ after PD and $47 \%$ after EC $(P=0.011)$. Major morbidity was
\end{abstract}

E. Melloul · N. Demartines ( $₫)$ · J.-M. Calmes · M. Schäfer Department of Visceral Surgery and Transplantation, University Hospital of Lausanne (CHUV), Lausanne, Switzerland e-mail: nicolas.demartines@chuv.ch

A. Denys

Department of Imaging and Interventional Radiology, University Hospital of Lausanne, 1011 Lausanne, Switzerland
$0 \%$ after PD and $21 \%$ after EC $(P=0.034)$. The mortality rate was not different (13\% after PD and $16 \%$ after EC, $P=1.0)$ and the deaths were all related to the patients' preexisting disease. Hospital and ICU stays were not different. Recurrent symptoms (17\%) occurred only after ACC in the PD group.

Conclusions In high-risk patients, PD and EC are both efficient in the resolution of acute cholecystitis sepsis. However, EC is associated with a higher procedure-related morbidity and the laparoscopic approach is not always possible. Percutaneous drainage represents a valuable intervention, but secondary cholecystectomy is mandatory in cases of acute calculous cholecystitis.

\section{Introduction}

Acute cholecystitis (AC) represents a very common surgical disease that is best treated by removal of the gallbladder. Most patients are admitted with acute calculous cholecystitis (ACC), whereas acute acalculous cholecystitis (AAC) occurs as a complication of severe preexisting diseases in critically ill patients [1,2]. During the last two decades, laparoscopic cholecystectomy has almost completely replaced the open approach, and it represents the current standard of treatment for acute and chronic cholecystitis [3, 4]. Even complicated cases under emergency conditions can be safely operated on laparoscopically [5]. While the vast majority of cases can be considered uncomplicated, some patients develop cholecystitis sepsis that requires intensive care medicine and distinct treatment options taking into account the patient's poor general condition. In particular, patients with AAC have markedly increased mortality rates ranging from 22 to $71 \%$ [1, 2]. 
In order to avoid the surgical trauma of a cholecystectomy and its related morbidity in critically ill patients, different conservative and interventional strategies have been developed recently. One such strategy is percutaneous drainage (PD) of the gallbladder to remove the infected bile as the infectious source of biliary sepsis.

Since first reported in 1980 [6], several series have demonstrated the feasibility of PD in high-risk patients with AC-related sepsis [7-21]. However, there are only scarce data comparing PD to emergency surgery, i.e., laparoscopic cholecystectomy. This lack of data is related to various factors. There is no generally accepted definition of high-risk patients. Often, age or the American Society of Anesthesiology (ASA) score is used as a surrogate parameter instead of a precise assessment of organ failure to define critical illness. In addition, many surgeons and anesthetists still prefer open cholecystectomy in high-risk patients. Finally, biliary sepsis caused by AC in critically ill patients is a rather rare event, and randomization of patients may probably never be achieved.

The aim of the current study was to compare PD of the gallbladder to emergency cholecystectomy (EC) in a welldefined patient group with biliary sepsis related to AC after failed conservative treatment. Procedure-related morbidity and mortality as well as long-term outcome were of primary interest.

\section{Patients and methods}

\section{Patients}

From January 2001 to December 2007, PD or EC was performed in 42 patients with biliary sepsis caused by ACC and AAC. All patients were critically ill according to the definitions used as mentioned below, and treatment in the ICU at the University Hospital of Lausanne (Switzerland) was mandatory for resuscitation. Patients with additional cholangitis or pancreatitis were excluded.

Patients were identified from prospective databases of the Departments of Intensive Care Medicine and Visceral Surgery. Their medical charts and radiological and pathological reports were carefully reviewed to extract data and outcome parameters. Data were documented on a separate data sheet developed a priori for each patient.

The severity of disease at the time of cholecystitis sepsis was assessed using the Simplified Acute Physiology Score II (SAPS II) [22-24]. This score includes the type of admission (medical or surgical), preexisting chronic disease, Glasgow coma scale, age, blood pressure, heart rate, fever, urine output, white blood cell count (WBC), electrolytes values, bilirubin level as parameters; it is a wellaccepted and well-used risk score for ICU patients. As
SAPS scores of 15 or greater are associated with a high predictive mortality rate in surgical ICU patients [25], we used this value as a cutoff to define high-risk patients.

Diagnosis of acute cholecystitis and sepsis

The diagnosis of AC was based on the patient's history, clinical signs (i.e., positive Murphy's sign, fever, pain in the right upper quadrant), laboratory finding (CRP > $10 \mathrm{mg} / \mathrm{l}, \mathrm{WBC}>10 \mathrm{~g} / \mathrm{l})$, and positive computed tomography (CT) and/or ultrasound (US) imaging. Acute cholecystitis was considered on imaging if there was greater than $10-\mathrm{cm}$ enlargement of the anteroposterior gallbladder axis and gallbladder wall thickening greater than $3 \mathrm{~mm}$. The diagnosis of gangrenous cholecystitis (GC) was based on histopathological analysis of the gallbladder.

Sepsis was defined as infection causing hypotension that requires colloids or catecholamines supports, and/or with one or more dysfunctional organs [26].

\section{Assessment of organ dysfunction}

The sequential organ failure assessment (SOFA) score was used to assess the severity of organ dysfunction in all patients with sepsis caused by AC. In order to document the clinical course, organ dysfunction was assessed at the time of diagnosis of AC, 3 and 7 days after PD or EC. The SOFA score is a validated score that is widely used to describe organ dysfunction/failure in critically ill patients [27, 28]. It includes cardiovascular, renal, hepatic, respiratory, and neurologic parameters [27, 28].

\section{Treatment algorithm (Fig. 1)}

All patients admitted with sepsis caused by ACC or AAC were seen primarily by the surgeon or internist on call. Critically ill patients were immediately transferred to the ICU and treatment was started by using standard resuscitation with fluid/catecholamine support and broad-spectrum antibiotics. For critically ill patients who developed AC sepsis while in the ICU for other pathologies, the surgeon on call was always involved in the primary assessment of the patient. ICU patients were regularly reviewed by an interdisciplinary team, including surgeons, intensivists, and, if necessary, interventional radiologists and anesthesiologists. If there was no clinical improvement within 12-24 h, EC or interventional gallbladder drainage was discussed within the interdisciplinary team. The final decision on the type of intervention to use was made by the senior surgeon on call based on the patient's condition and the availability of interventional radiology within $6 \mathrm{~h}$. 
Fig. 1 Treatment algorithm

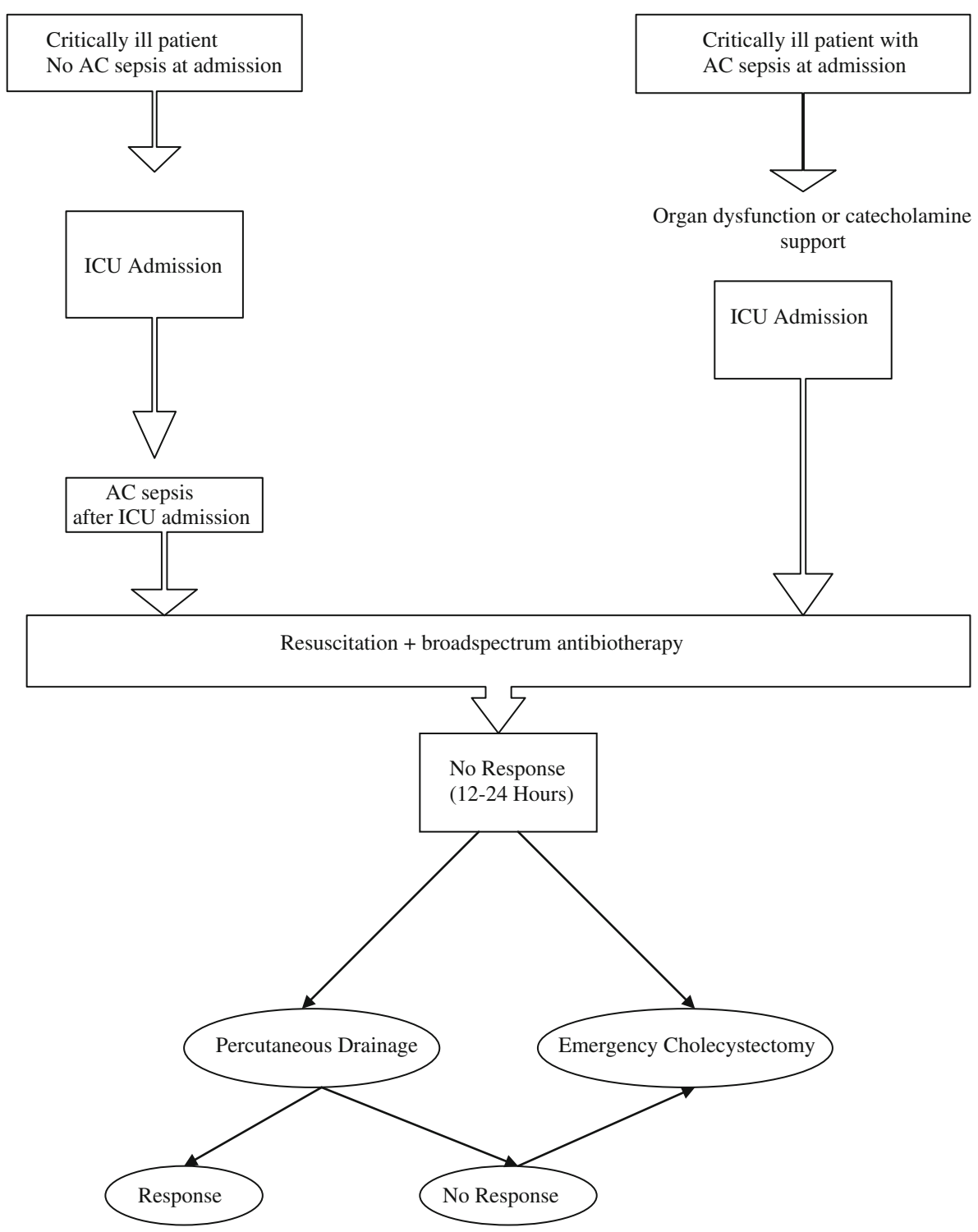

Percutaneous drainage of the gallbladder

Unless patients were already intubated, all procedures were performed under local anesthesia. Biliary tract imaging and interventions were done on a dedicated system in a standard fashion. Following percutaneous transhepatic puncture of the gallbladder under US or CT guidance, an 8-Fr Autolock catheter (Boston Scientific, Natick, MA, USA) was inserted using the standard Seldinger technique.

Complications related to the procedure were evaluated using the Society of Interventional Radiology classification system for complications by outcome [29]. Major complications were defined as any procedure-related morbidity requiring either radiological or emergency surgical intervention, or death of a patient. Mortality was defined as any death that occurred during the PD procedure up to 90 days after hospital admission.
Positive response to $\mathrm{PD}$ was defined as improvement in clinical signs (reduction of abdominal tenderness, fever, and, in the case of severe sepsis, of catecholamine perfusion) and/or organ dysfunction within $12 \mathrm{~h}$ after the procedure. If these criteria were not met and provided that no other cause of sepsis was found, EC was mandatory to treat PD failure. The criteria for catheter removal were resolution of sepsis and a patent cystic duct after transcatheter cholecystography [30]. After drain removal, the transhepatic channel was occluded with a hemostatic device (Gelfoam, Pfizer) in all cases.

\section{Emergency cholecystectomy}

Laparoscopic cholecystectomy was always attempted if there were no contraindications for a minimally invasive approach. While open cholecystectomy was performed by 
using a subcostal incision, a 4-trocar technique was the standard approach for a laparoscopic cholecystectomy. In all cases, the gallbladder was removed in toto, i.e., no subtotal cholecystectomy was performed. Intraoperative cholangiography was only selectively performed. Specimens were sent for routine pathological examination.

Complications related to surgery were assessed using a validated classification score [31]. Major complications were classified as grade III-V and referred to any lifethreatening morbidity requiring either surgical or radiological intervention or patient death. Mortality was defined as any death occurring during the surgical procedure up to 90 days after hospital admission. All surgical interventions were performed and supervised by one of the five senior staff surgeons.

\section{Statistics}

Categorical values were expressed as percentage. Continuous variables were expressed as mean, standard deviation, median, and range. Fisher's exact test and Wilcoxon's test were used for statistical evaluation, and $P<0.05$ was considered statistically significant.

\section{Results}

Patients

There were 34 men and 8 women [median age $=65.5$ years (range $=32-94$ )] included in the current study. While $19(45 \%)$ patients underwent EC, the remaining 23 $(55 \%)$ patients had interventional PD of the gallbladder. Both patient groups had similar characteristics, particularly age, SAPS scores, and preexisting comorbidities (Table 1).

Although sepsis caused by $\mathrm{AC}$ was the main reason for admission to the ICU for 11 of 23 patients with PD (48\%) and for 9 of 19 patients with $\mathrm{EC} \mathrm{(47 \% ),} \mathrm{the} \mathrm{remaining}$ patients developed AAC or ACC in the ICU as a complication of an underlying disease (Table 2). The initial median SOFA scores at the time of cholecystitis sepsis diagnosis were 4.5 (range $=1-15$ ) for the PD group and 8 (range $=1-18$ ) for the EC group, with no statistically significant difference $(P=0.29)$.

Interventional versus surgical treatment

In accordance with our treatment algorithm, percutaneous gallbladder drainage was technically successful in all 23 patients who were scheduled for PD. In 21 of 23 patients (91\%), cholecystitis sepsis rapidly resolved, with clinical improvement within $24 \mathrm{~h}$ and improvement in organ dysfunction within 3 days as reflected by significantly reduced
Table 1 Preoperative characteristics of patients

\begin{tabular}{llll}
\hline & PD group & EC group & $P$ \\
\hline$N$ & 23 & 19 & \\
Age (years) & $65(37-86)^{\mathrm{a}}$ & $63(32-85)^{\mathrm{a}}$ & 0.85 \\
Male/female $(n)$ & $18 / 5$ & $16 / 3$ & 0.53 \\
ACC/AAC $(n)$ & $11 / 12$ & $13 / 6$ & 0.22 \\
SAPS II score at the time of & $34(15-71)^{\mathrm{a}}$ & $31(15-72)^{\mathrm{a}}$ & 0.55 \\
$\quad$ cholecystitis diagnosis & & & \\
Comorbidities $(n)$ & 47 & 24 & \\
Cardiomyopathy & 12 & 5 & 0.12 \\
Renal insufficiency & 5 & 5 & 0.25 \\
Diabetes & 8 & 2 & 0.08 \\
Obesity & 6 & 2 & 0.26 \\
COPD & 6 & 3 & 0.43 \\
Hypertension & 10 & 7 & 0.34 \\
\hline$A C C / A A C$ acute cal & & & \\
\hline
\end{tabular}

$\overline{A C C / A A C \text { acute calculous cholecystitis/acute acalculous cholecysti- }}$ tis, SAPS simplified acute physiology score

${ }^{a}$ Median values (range)

Table 2 Indications for ICU admission

\begin{tabular}{lll}
\hline & $\begin{array}{l}\text { PD group } \\
(n=23)\end{array}$ & $\begin{array}{l}\text { EC group } \\
(n=19)\end{array}$ \\
\hline Acute cholecystitis sepsis & 11 & 9 \\
Cardiac failure & 4 & 1 \\
Abdominal trauma & 2 & 3 \\
Others $^{\text {a }}$ & 6 & 6 \\
\hline
\end{tabular}

${ }^{a}$ Urosepsis, lung carcinoma, agranulocytosis, post esophagectomy, cervical fasciitis, post pulmonary lobectomy, post abdominal aortic aneurysm replacement, post lung transplantation

SOFA scores (Fig. 2). In two patients with ACC (9\%), interventional treatment failed and EC was mandatory. Both patients underwent laparoscopic cholecystectomy with an uneventful postoperative course. Histopathological assessment revealed GC in both cases. Overall, drains were removed after a median time of 7 days (range $=3-21$ days), and two patients were discharged at home with the catheter in place.

While ten patients underwent laparoscopic cholecystectomy, there were another nine patients who had primary open cholecystectomy. The indications for a primary open approach in that latter group were previous abdominal surgery $(n=2)$, trauma patients $(n=3)$, septic shock $(n=2)$, an unclear mass in the pancreatic head $(n=1)$, and severe heart failure with extracorporeal cardiac assistance $(n=1)$. Three patients in the laparoscopic group were converted to open (conversion rate $=16 \%$ ) due to severe adhesions impairing adequate visualization and extreme friability of tissues making further dissection hazardous. Biliary sepsis was resolved in all patients and 


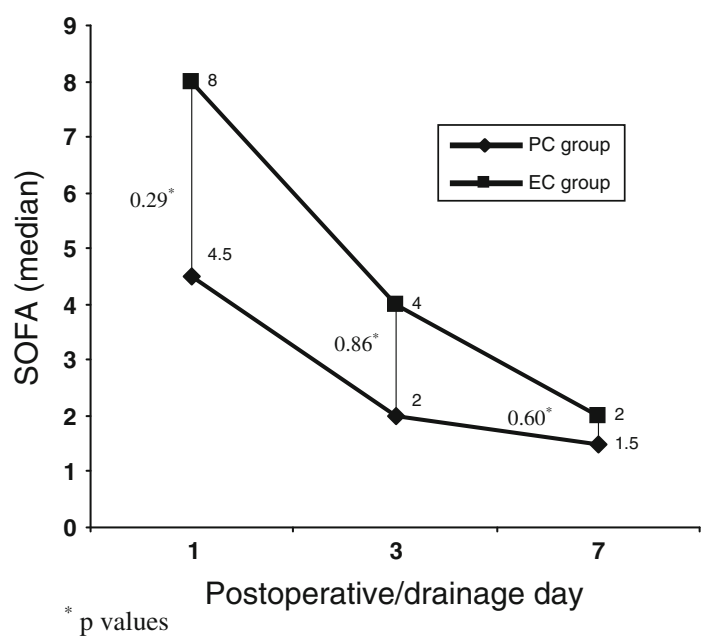

Fig. 2 Evolution of SOFA (Sequential Organ Failure Assessment) score at the time of acute cholecystitis diagnosis (day 1) and days 3 and 7 after $\mathrm{PC}$ or $\mathrm{EC} ; * P$ values

organ dysfunction improved after 3 days as shown by decreased SOFA scores (Fig. 2).

Complication rates, mortality, and length of hospital stay (Table 3)

The overall complication rate was $8.7 \%(2 / 23)$ after PD and $47 \%(9 / 19)$ after EC $(P=0.011)$. There were no major complications related to PD. Minor complications consisted of one drain dislodgement and one abdominal wall hematoma. In the EC group, minor complications (grades I and II) included two paralytic ileus, two abdominal wall abscesses, and one cholangitis. Major complications (grades III-V) occurred in 21\% (4/19) after EC. Severe bleeding from the gallbladder bed and high-output biliary leak in two patients each required open reoperation at postoperative day 1 .

The overall 90-day mortality rate was $13 \%(3 / 23)$ after PD and $16 \%(3 / 19)$ after EC $(P=1.0)$. In the PD group,

Table 3 Complication rates, mortality, and length of hospital stay

\begin{tabular}{llll}
\hline & PD group & EC group & $P$ \\
\hline$N$ & 23 & 19 & \\
Overall complication rate $^{\text {a }}$ & $2(8.7 \%)$ & $9(47 \%)$ & 0.011 \\
Minor complications $^{\text {a }}$ & $2(8.7 \%)$ & $5(26 \%)$ & 0.21 \\
Major complications $^{\text {a,b }}$ & 0 & $4(21 \%)$ & 0.03 \\
90-day mortality & $3(13 \%)$ & $3(16 \%)$ & 1.0 \\
Overall hospital stay in days $_{\text {ICU stay in days }}$ & $25(7-97)$ & $23(5-65)$ & 0.39 \\
\hline
\end{tabular}

a According to the SIR classification for PD and Dindo-Clavien classification for EC group

${ }^{\mathrm{b}}$ Grade III-V according to the Dindo-Clavien classification for the EC group acute cardiac failure, stroke, and agranulocytosis with multiple organ failure in the context of a myeloma were the reasons for the postoperative deaths. In the EC group, multiple organ failure in the context of a myeloma, ischemic colitis secondary to an operated ruptured abdominal aortic aneurysm, and a duodenal fistula after an esophagectomy were the causes of death.

Mean ICU stay was 10.5 days (range $=2-71$ days) and 3 days (range $=2-31$ days) for the PD and EC group, respectively. Overall, the length of hospital stay was 25 days (range $=7-97$ days) in the PD group and 23 days (range $=5-65$ days) in the EC group. Neither length of ICU stay nor overall length of hospital stay were significantly different for the treatment groups $(P=0.17$ and 0.39 ).

\section{Long-term follow-up}

Four patients after primary successful percutaneous gallbladder drainage developed recurrent biliary symptoms during long-term follow-up (median followup $=16$ months, range $=2-44$ months). These symptoms included recurrent right-upper-quadrant pain $(n=2)$ and de novo AC without sepsis $(n=2)$. All four patients had previous ACC, whereby two patients revealed an impacted gallstone in the gallbladder infundibulum causing a cystic duct obstruction and the drain was left after hospital discharge. One patient underwent laparoscopic cholecystectomy 14 days after hospital discharge due to drain dislodgement; two other patients were treated successfully by laparoscopic cholecystectomy at the time of recurrence ( 2 and 12 months after hospital discharge). The final patient denied surgery after drain removal and was lost during the follow-up.

\section{Discussion}

This current study assessed the role of interventional percutaneous gallbladder drainage compared to EC for the treatment of AC sepsis in critically ill patients. To this end, 42 patients of a single center were retrospectively analyzed during a 7-year period. Both PD and EC were able to resolve quickly cholecystitis sepsis in $90-100 \%$ of the patients. Surgery was associated with an increased complication rate of $47 \%$ compared to interventional treatment (8.7\%), but mortality rates did not show any difference.

The optimal treatment of AC with concomitant sepsis in critically ill patients has not yet been well defined. In particular, there is only limited evidence in the literature about whether PD or surgical removal of the gallbladder should be preferred. Both approaches are currently used, and specific indications on which approach to use are 
mostly dependent on local experience and personal preferences. In addition, with the successful advance of laparoscopic surgery and modern interventional radiology, the role of open cholecystectomy has been increasingly challenged.

Percutaneous drainage of the gallbladder has been developed as a less invasive procedure compared to open cholecystectomy in critically ill patients with AC. From a technical point of view, it is a rather uncomplicated procedure with a low complication rate that is reported to range from 3 to $13 \%$ [7-21, 32, 33]. The complication rate in this current series was $8.7 \%$ with no major complication. This low complication rate is related to the fact that only experienced radiologists performed all the interventional procedures and intrahepatic tracts were systematically occluded after drain removal to prevent biliary leaks and bleeding. Mortality after PD was $13 \%$, which did not differ from that of EC (16\%) and the reported mortality in the literature that ranges from 0 to $11 \%$ (see Table 4). Of note, mortality is predominantly related to the severity of the underlying disease rather than the ongoing gallbladder sepsis. Those latter cases have to be considered as a failure of interventional treatment, and EC is mandatory. PD successfully resolved sepsis in all but two patients (success rate $=91 \%$ ) in our series, which is within reported clinical success rates of $78-100 \%$ (Table 4). Both patients who needed an EC as a salvage procedure revealed GC. Similar findings have been already reported; thus, GC that occurs in $40-80 \%$ of patients with AAC and in $2-31 \%$ of patients with ACC [2, 20, 34-38] seems to be an important risk factor for PD to fail. It can therefore be assumed that the underlying diseases are more important for long-term survival than the type of intervention.

While relief of sepsis could be achieved in $90 \%$ of patients, four patients with ACC (17\%) developed recurrent biliary symptoms during the long-term follow-up which required laparoscopic cholecystectomy. It has been reported recently that up to $40 \%$ of patients treated with PD needed secondary cholecystectomy, whereby the mortality rate was below 2\% [33]. Welschbilig-Meunier et al. [20] promote prophylactic cholecystectomy only in patients drained for ACC. Our results seem to support that; however, our data lack statistical power to be affirmative. Therefore, primary cholecystectomy or scheduled resection after drainage is recommended to prevent biliary complications in the long-term course, particularly after ACC.

A laparoscopic attempt to remove the gallbladder was made in only ten patients $(52 \%)$, and nine patients were considered for primary open cholecystectomy. Thus, open cholecystectomy has not yet replaced laparoscopy since patients with biliary sepsis requiring ICU treatment often are too sick. Moreover, intraoperative conversion to open cholecystectomy was needed in two patients $(16 \%)$ due to inflammatory changes that impaired safe identification of biliary and vascular anatomy in the hepatic pedicle. Since EC is a life-saving procedure, an increased complication rate of $47 \%$ is probably to be expected. Nevertheless, all major complications, i.e., bleeding and biliary leaks,

Table 4 Review of the literature

\begin{tabular}{|c|c|c|c|c|c|c|c|c|}
\hline & $\begin{array}{l}\text { Year of } \\
\text { publication }\end{array}$ & $N$ & $\begin{array}{l}\text { Mean age } \\
\text { (years) }\end{array}$ & $\begin{array}{l}\text { Calculous/ } \\
\text { Acalculous }\end{array}$ & $\begin{array}{l}\text { PD } \\
\text { success }(\%)\end{array}$ & $\begin{array}{l}\text { 90-days mortality } \\
\text { due to } \mathrm{AC}^{\mathrm{a}}(\%)\end{array}$ & $\begin{array}{l}\text { Recurrent } \\
\text { symptoms (\%) }\end{array}$ & $\mathrm{EC}(\%)$ \\
\hline Sugiyama et al. [7] & 1998 & 38 & 85 & $25 / 13$ & 95 & $?$ & 33 & 0 \\
\hline Davis et al. [8] & 1999 & 22 & 77 & $14 / 6$ & 82 & $?$ & 9 & 0 \\
\hline Ghahreman et al. [9] & 1999 & 19 & 70 & $14 / 4$ & 78 & 0 & 5 & 0 \\
\hline Patel et al. [10] & 2000 & 53 & 63 & $39 / 14$ & 83 & 11 & 0 & 0 \\
\hline Pessaux et al. [11] & 2000 & 29 & 80 & $21 / 8$ & 86 & 0 & 7 & 14 \\
\hline Chang et al. [12] & 2000 & 24 & 65 & $16 / 7$ & 83 & 4 & 8 & 0 \\
\hline Hatzidakis et al. [13] & 2002 & 63 & 79 & $44 / 19$ & 86 & 9.5 & 2 & 11 \\
\hline Spira et al. [14] & 2002 & 55 & 74 & $?$ & 98 & 0 & 18 & 4 \\
\hline Andren-Sandberg et al. [15] & 2001 & 86 & 71 & $?$ & 89 & 6 & 32 & 1 \\
\hline Granlund et al [16] & 2001 & 51 & 71 & $28 / 23$ & 90 & 0 & 21 & 2 \\
\hline Berman et al. [17] & 2002 & 10 & 77 & $10 / 0$ & 100 & 0 & $?$ & 0 \\
\hline Byrne et al. [18] & 2003 & 45 & 63 & $?$ & 78 & 2 & 2 & 0 \\
\hline Li et al. [19] & 2004 & 25 & 81 & $?$ & 92 & 0 & 12 & 8 \\
\hline Welschbilig-Meunier et al. [20] & 2005 & 65 & 78 & $49 / 16$ & 90 & 1.5 & 25 & 3 \\
\hline Ha et al. [21] & 2008 & 65 & 63 & $65 / 0$ & 91 & $?$ & 35 & $?$ \\
\hline Present study & 2010 & 23 & 65 & $11 / 12$ & 91 & 0 & 19 & 8.7 \\
\hline
\end{tabular}

${ }^{a}$ Acute cholecystitis (not confirmed by autopsy) 
required early open reoperation. Gangrenous cholecystitis was found in four patients, three of whom underwent primary open cholecystectomy and one had a laparoscopic cholecystectomy that was converted to an open. Since the source of the biliary sepsis is removed, all patients improved clinically and no recurrent biliary symptoms occurred in the long term. Mortality after EC was 16\%, but as in the PD group, it almost always was related to the patients' underlying diseases.

In conclusion, PD and EC are highly efficient in resolving cholecystitis sepsis in critically ill patients with severe AC. While mortality rates are similar, surgery is associated with significantly increased perioperative morbidity. Furthermore, a laparoscopic approach is not always possible. Percutaneous drainage represents a valuable intervention to treat cholecystitis sepsis, but it should be completed by a secondary cholecystectomy, particularly in patients with ACC.

\section{References}

1. Kalliafas S, Ziegler D, Flancbaum L et al (1998) Acute acalculous cholecystitis. Incidence, risk factors, diagnosis, and outcome. Am Surg 64:471-475

2. Laurila J, Syrjälä H, Laurila PA et al (2004) Acute acalculous cholecystitis in critically ill patients. Acta Anaesthesiol Scand 48:986-991

3. Cushieri A, Dubois F, Mouiel J et al (1991) The European experience with laparoscopic cholecystectomy. Am J Surg 161:385-387

4. Wiseman JT, Sharuk MN, Singla A et al (2010) Surgical management of acute cholecystitis at a tertiary care center in the modern era. Arch Surg 145:439-444

5. Gurusamy K, Samraj K, Gluud C et al (2010) Meta-analysis of randomized controlled trials on the safety and effectiveness of early versus delayed laparoscopic cholecystectomy for acute cholecystitis. Br J Surg 97:141-150

6. Radder RW (1980) Ultrasonography guided percutaneous catheter drainage for gallbladder empyema. Diagn Imaging 49: 330-333

7. Sugiyama M, Tokuhara M, Atomi Y (1998) Is percutaneous cholecystostomy the optimal treatment for acute cholecystitis in the very elderly? World J Surg 22:459-463

8. Davis CA, Landercasper J, Gundersen LH et al (1999) Effective use of percutaneous cholecystostomy in high-risk surgical patients. Arch Surg 134:727-732

9. Ghahreman A, McCall JL, Windsor JA (1999) Cholecystostomy: a review of recent experience. Aust N Z J Surg 69:837-840

10. Patel M, Miedema BW, James MA et al (2000) Percutaneous cholecystostomy is an effective treatment for high-risk patients with acute cholecystitis. Am Surg 66:33-37

11. Pessaux P, Tuech JJ, Regenet N et al (2000) Laparoscopic cholecystectomy in acute cholecystitis. A prospective comparative study in patients with acute vs. chronic cholecystitis. Surg Endosc $14: 358-361$

12. Chang L, Moonka R, Stelzner M (2000) Percutaneous cholecystostomy for acute cholecystitis in Veteran patients. Am J Surg 150:198-202
13. Hatzidakis AA, Prassopoulos P, Petinarakis I et al (2002) Acute cholecystitis in high-risk patients: percutaneous cholecystostomy vs conservative treatment. Eur Radiol 12(7):1778-1784

14. Spira RM, Nissan A, Zamir O et al (2002) Percutaneous cholecystostomy and delayed laparoscopic cholecystectomy in critically ill patients with acute calculous cholecystitis. Am J Surg 183:62-66

15. Andren-Sandberg A, Haugsvedt T, Larssen TB et al (2001) Complications and late outcome following percutaneous drainage of the gallbladder in acute calculous cholecystitis. Dig Surg 18:393-398

16. Granlund A, Karlson BM, Elvin A et al (2001) Ultrasound guided percutaneous cholecystostomy in high risk surgical patients. Langenbeck's Arch Surg 386:212-217

17. Berman M, Nudelman IL, Fuko $Z$ et al (2002) Percutaneous transhepatic cholecystostomy: effective treatment of acute cholecystitis in high risk patients. Isr Med Assoc J 4:331-333

18. Byrne MF, Suhocki P, Mitchell MR et al (2003) Percutaneous cholecystostomy in patients with acute cholecystitis: Experience of 45 patients at a US referral center. J Am Coll Surg 197:206-211

19. Li JCM, Lee DWH, Lai CW et al (2004) Percutaneous cholecystostomy for the treatment of acute cholecystitis in the critically ill and elderly. Hong Kong Med J 10(6):389-393

20. Welschbilig-Meunier K, Pessaux P, Lebigot J et al (2005) Percutaneous cholecystostomy for high-risk patients with acute cholecystitis. Surg Endosc 19:1256-1259

21. Ha JP, Tsui KK, Tang CN et al (2008) Cholecystectomy or not after percutaneous cholecystostomy for acute calculous cholecystitis in high-risk patients. Hepatogastroenterology 55(86-87): 1497-1502

22. Le Gall JR, Lemeshow S, Saulnier F (1993) A new simplified acute physiology score (SAPS II) based on a European/North American multicenter study. JAMA 270:2957-2963

23. Rosenberg AL (2002) Recent innovations in intensive care unit risk-prediction models. Curr Opin Crit Care 8:321-333

24. Den Boer S, De Keizer NF, De Jonge E (2005) Performance of prognostic models in critically ill cancer patients-a review. Crit Care 9(4):458-463

25. Knaus WA, Draper EA, Wagner DP et al (1985) APACHE II: a severity of disease classification system. Crit Care Med 13: 818-829

26. Lever A, Mackenzie I (2007) Sepsis: definition, epidemiology, and diagnosis. BMJ 335:879-883

27. Vincent JL, Moreno R, Takala J et al (1996) The SOFA (Sepsis related Organ Failure Assessment) score to describe organ dysfunction/failure. Intensive Care Med 22:707-710

28. Vincent JL, De Mendonca A, Cantraine F et al (1998) Use of the SOFA score to assess the incidence of organ dysfunction/failure in intensive care units: results of a multicenter prospective study. Crit Care Med 26:1793-1800

29. Sacks D, McClenny TE, Cardella JF et al (2003) Society of interventional radiology clinical practice guidelines. J Vasc Interv Radiol 14:S199-S202

30. Vauthey JN, Lerut J, Martini M et al (1993) Indications and limitations of percutaneous cholecystostomy for acute cholecystitis. Surg Gynecol Obstet 176(1):49-54

31. Dindo D, Demartines N, Clavien PA (2004) Classification of surgical complications: a new proposal with evaluation in a cohort of 6336 patients and results of a survey. Ann Surg 240:205-213

32. Ginat D, Saad WE (2008) Cholecystostomy and transcholecystic biliary access. Tech Vasc Interv Radiol 11(1):2-13

33. Winbladh A, Gullstrand P, Svanvik J et al (2009) Systematic review of cholecystostomy as a treatment option in acute cholecystitis. HPB (Oxford) 11(3):183-193 
34. Eldar S, Sabo E, Nash E et al (1998) Laparoscopic cholecystectomy for the various types of gallbladder inflammation, a prospective trial. Surg Laparosc Endosc 8:200-207

35. Singer JA, Mckeen RV (1994) Laparoscopic cholecystectomy for acute or gangrenous cholecystitis. Am Surg 60:326-328

36. Wilson AK, Koszol RA, Salwen WA et al (1994) Gangrenous cholecystitis in an urban VA hospital. J Surg Res 56:402-404
37. Merriam LT, Kanaan SA, Dawes LC et al (1999) Gangrenous cholecystitis: analysis of risk factors and experience with laparoscopic cholecystectomy. Surgery 126:680-686

38. Boggi U, Di Candio G, Campatelli A et al (1999) Percutaneous cholecystostomy for acute cholecystitis in critically ill patients. Hepatogastroenterology 46:121-125 